\title{
Cómo manejar el comportamiento irracional de los demás
}

How to handle the irrational behavior of others

\author{
Gema Torrens Espinosa \\ Universitat de les Illes Balears \\ gema.torrens@uib.es
}

\section{Resumen}

Todos mantenemos actitudes irracionales en algún momento, aunque no solemos darnos cuenta pues nos resulta más obvio detectar estos comportamientos en los demás.

Ciertamente, en algunas persones estas actitudes problemáticas son tan frecuentes, intensas y duraderas que se pueden considerar como persones "difíciles". Para puestos de trabajo de atención al usuario, como lo es el de Defensor Universitario, se hace necesario normalizar que un porcentaje elevado de nuestros consultantes presentan este perfil y, por este motivo, necesario es también adquirir recursos para su manejo.

Este artículo pretende ofrecer datos de prevalencia sobre Trastornos Mentales, así como herramientas de comunicación asertiva para manejar estas situaciones tensas.

Palabras clave: Comportamiento irracional, asertividad, análisis transaccional, habilidades sociales, gestión del conflicto.

\section{Abstract}

We all maintain irrational attitudes at some point, although we do not usually realize it because it is more obvious for us to detect these behaviors in others.

Certainly, in some people these problematic attitudes are so frequent, intense and long-lasting that they can be considered as "difficult" people. For user service jobs, such as University Defender, it is necessary to normalize that a high percentage of our consultants present this profile and, for this reason, it is also necessary to acquire resources for its management.

This article aims to offer prevalence data on Mental Disorders, as well as assertive communication tools to handle these tense situations.

Keywords: Irrational behavior, assertiveness, transactional analysis, social skills, conflict management.

\section{Sumario}

introducción. 1-Prevalencia de los trastornos mentales. 2-Manejar el comportamiento irracional de los demás. 3-Conclusiones. 4-Referencias. 


\section{Introducción}

El pasado 17 de junio tuvo lugar una webinar dentro del marco de la I Jornada Técnica de Defensores Universitarios (CEDU) que se había previsto para el 8 de mayo antes de que se decretara el estado de alarma. La propuesta, en principio, era elaborar dos talleres sobre habilidades y estrategias para afrontar las situaciones relacionadas o producidas por personas con problemas de salud mental.

Ante la imposibilidad de llevar a cabo dicha Jornada, y viendo la necesidad de analizar lo que nos encontraríamos después del confinamiento, se decidió llevar a cabo esta webinar con el siguiente contenido:

\section{¿Qué nos espera a la vuelta?}

1. Trabajo, Conciliación y Covid-19: algunos datos.

2. La Entrevista: herramientas para afrontarla.

3. Cómo manejar el comportamiento irracional del otro/a.

El primer punto corresponde a un estudio elaborado por un equipo de investigación de la UIB realizado en diferentes tiempos durante el confinamiento sobre emociones relacionadas con la situación COVID y sobre teletrabajo y conciliación. Esta parte la desarrolló la Dra. Esther García Buades, del área de Psicología Social de la UIB.

En el segundo punto se dieron pautas para afrontar una entrevista y se dieron técnicas de comunicación asertiva para ello. Esta parte la desarrolló la Dra. Carmen Ramis Palmer, del área de Psicología Social de la UIB.

En el tercer punto se dieron herramientas para manejar el comportamiento irracional de la otra persona. Este artículo corresponde a este tercer punto que desarrolló la profesora Gema Torrens Espinosa.

\section{Prevalencia de los trastornos mentales}

Según el estudio ESEMeD-España de la Unidad de Investigación y Desarrollo del Servicio de Salud Mental del Hospital San Joan de Déu de Barcelona y de la Unidad de Investigación en Servicios Sanitarios del Instituto Municipal de Investigación Médica de Barcelona, un $19,5 \%$ de las personas presentaron un trastorno mental en algún momento de su vida (prevalencia-vida). En conclusión, podemos afirmar que casi 1 de cada 5 personas presenta un trastorno mental a lo largo de su vida. En este porcentaje se incluyen la Depresión Mayor y los Trastornos de Ansiedad, que son los más prevalentes, así como los trastornos de ansiedad y los inducidos por sustancias químicas, etc.

Es altamente probable que a la Oficina del Defensor Universitario acudan «personas difíciles»: personas irascibles con ira habitual, personas pasivo-agresivas, personas manipuladoras, maltratadoras, tramposas, críticas, comodonas, victimistas, personas con adicciones... Muchas de ellas con algún trastorno de personalidad que, con mucha probabilidad, no han sido diagnosticadas, y cuya prevalencia se encuentra entre el $4.4 \% \mathrm{y}$ el 19\% (Zaragoza et al., 2015) en la población normal. 


\section{1. Tipología de los trastornos de personalidad}

Podemos clasificar la tipología de los trastornos de personalidad en 3 grandes grupos:

Grupo A: personas que se muestran frías, retraídas, impacientes o irracionales.

- Trastorno paranoide de la personalidad. Es un patrón de desconfianza y suspicacia que hace que interpreten maliciosamente las intenciones de los demás.

- Trastorno esquizoide de la personalidad. Es un patrón de desconexión de las relaciones sociales y de restricción de la experiencia emocional.

- Trastorno esquizotípico de la personalidad. Es un patrón de malestar intenso en las relaciones personales, distorsiones cognitivas y perceptivas y de comportamiento excéntrico.

Grupo B: personas que transgreden las normas sociales, son impulsivas, muy emocionales, etc.

- Trastorno límite de la personalidad. Patrón de inestabilidad en las relaciones interpersonales, la autoimagen y los afectos, y de una notable impulsividad.

- Trastorno histriónico de la personalidad. Muy demandantes y de excesiva emotividad.

- Trastorno antisocial de la personalidad. Desprecian y violan los derechos de los demás.

- Trastorno narcisista de la personalidad. Patrón de grandiosidad, necesidad de admiración y falta de empatía.

Grupo C: personas que muestran miedo a las relaciones sociales, sumisas, etc.

- Trastorno de la personalidad por dependencia. Comportamiento sumiso y pegajoso relacionado con una excesiva necesidad de ser cuidado.

- Trastorno obsesivo-compulsivo de la personalidad. Excesiva preocupación por el orden, el perfeccionismo y el control.

- Trastorno de la personalidad por evitación. Patrón generalizado de inhibición social, sentimientos de inadecuación, hipersensibilidad a la evaluación negativa, rechazo o desaprobación, y evitación de la interacción social.

A excepción de los trastornos esquizoide y esquizotípico de la personalidad, es altamente probable que a la oficina acudan personas con estos trastornos mentales, además de Trastornos de Ansiedad, Depresión y trastornos inducidos por adicciones. Se hace necesario adquirir recursos para manejarlo.

\section{Manejar el comportamiento irracional de los demás}

\section{1. ¿Cómo manejamos habitualmente el comportamiento irracional de los demás?}

Necesitamos habilidades sociales para lidiar con las personas difíciles que acuden al servicio. Vicente Caballo, en su Manual de evaluación y entrenamiento de las 
habilidades sociales (1993), define las habilidades sociales como un "conjunto de conductas realizadas por un individuo en un contexto interpersonal que expresa sentimientos, actitudes, deseos, opiniones o derechos de un modo adecuado a la situación, respetando esas conductas en los demás, y que generalmente resuelve los problemas inmediatos de la situación mientras reduce la probabilidad de que aparezcan futuros problemas" (p. 407).

En su manual, Caballo expone 4 estilos de respuesta ante situaciones tensas:

1. Respuesta agresiva. Atacamos y rechazamos a la otra persona, pensando que "no tendrían que actuar así" y que "como actúan de esta manera son unos indeseables" (condena). Todo ello cargándonos de razones. En este estilo de respuesta, la emoción nos ha secuestrado y condiciona nuestro comportamiento, generalmente es rabia y/o indignación. Las consecuencias de tener este estilo son: perdemos credibilidad y reforzamos la actitud irracional del otro.

2. Respuesta pasivo-inhibida. Cedemos, pensando "he de tener paciencia y evitar problemas mayores que se darían si yo protesto o muestro rechazo por su conducta irracional". De este modo, perdemos el liderazgo de la conversación. En este estilo también estamos secuestrados por la emoción, en este caso miedo y ansiedad. Las consecuencias de tener este estilo son: No se resuelve el problema eficazmente, suele quedar pendiente por la evitación; la otra persona se crece y no aprende a respetarte; aparición de emociones negativas a largo plazo: ansiedad, ira, tristeza y baja autoestima.

3. Respuesta pasivo-agresiva. Respondemos como un pasivo porque no decimos nada directamente ni en el momento; actuamos como un agresivo: Castigamos a la persona con consecuencias negativas, de manera deshonesta y a destiempo. Esta dinámica puede ser consciente o no. En este caso la emoción que nos secuestra es la venganza, la rabia y/o la cobardía. Las consecuencias de tener este estilo son las mismas que en el estilo agresivo y se puede despertar el deseo de venganza del otro. es importante distinguir entre una reacción pasivo-agresiva, que todos podemos mostrar en algún momento, de un trastorno de personalidad pasivoagresivo, que consiste en este estilo como patrón de comportamiento y no como una reacción puntual y aislada.

4. Respuesta asertiva. En este estilo, buscamos el momento oportuno para hablar y la forma más adecuada de impedir que sigan molestándonos o interrumpiendo, no estamos secuestrados por la emoción. Respondemos de manera aséptica ofreciendo datos, información, sin entrar en personalismos. Diferenciamos entre el su comportamiento no deseable y la persona en sí misma, que es mucho más que una conducta.

Las personas tenemos los 4 estilos de respuesta aunque mostramos una tendencia.

¿Conocemos nuestra tendencia? Algunas reflexiones interesantes añadidas pueden ser:

¿Quién despierta tu lado más irracional? ¿Con quién no muestras tu tendencia?

¿Qué tiene esa persona? 


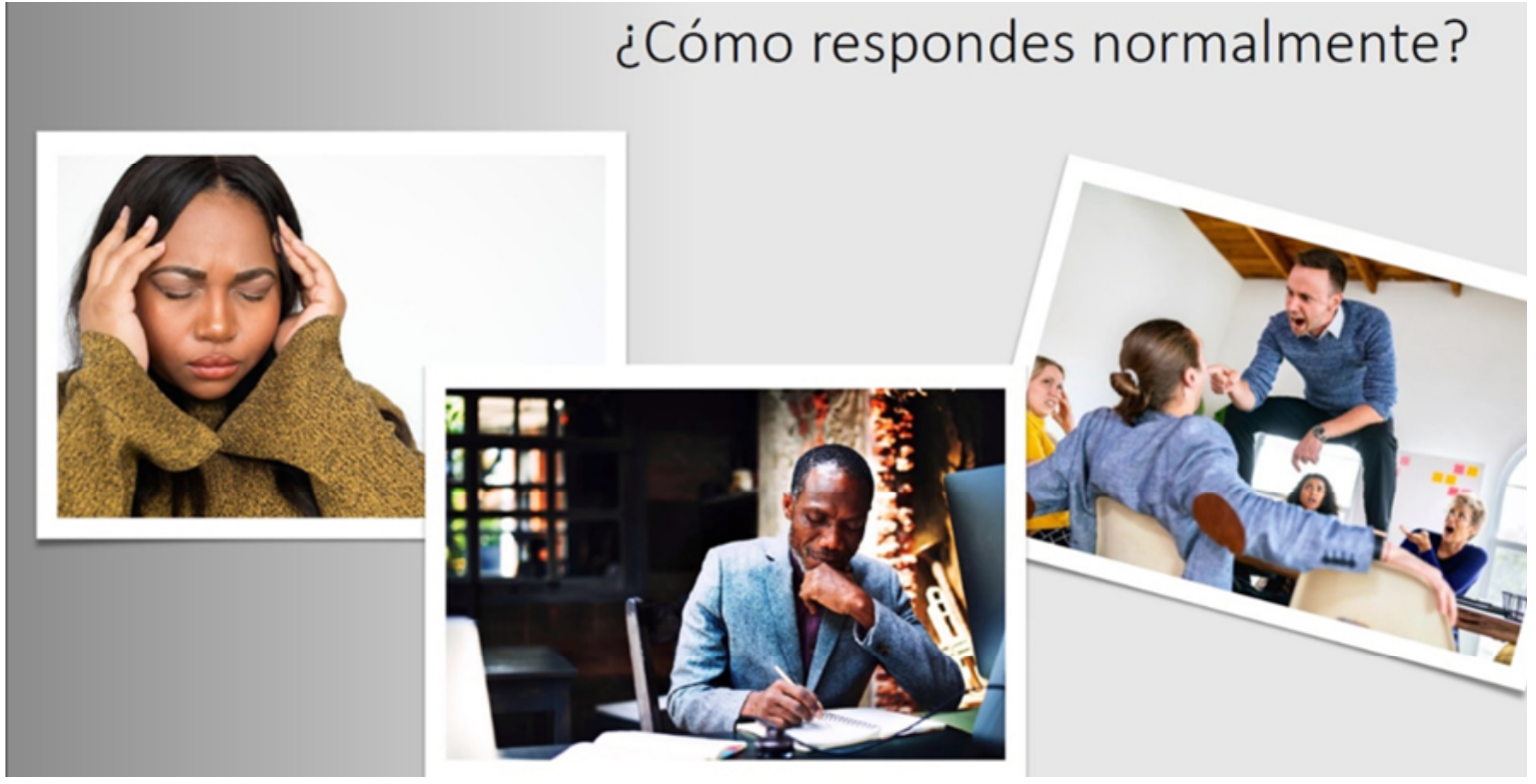

\subsection{Intervención en 4 pasos}

Cuanto más irracional es la otra persona, más racional me muestro yo. Requisito indispensable para ello es contar con herramientas de autorregulación emocional, que se pueden aprender, repito, si se entrenan.

1. PENSAR asertivamente y CUIDAR el lenguaje NO-VERBAL

- Cambiar condena y autocondena por derechos asertivos.

- Derecho a cometer errores (nosotros y el otro).

- Es normal sentirse incómodo ante lo irracional.

- Puedo responder de forma positiva.

\section{ESCUCHAR ATENTAMENTE Y PEDIR DETALLES}

3. Mostrar ACUERDO EN LO POSIBLE (Técnica banco de niebla): en todo, en parte, o en el derecho a verlo así.

\section{EXPRESAR NUESTRA OPINIÓN DE FORMA ASÉPTICA.}

- Si está de acuerdo, dígalo serenamente.

- Si lo está en parte: "Entiendo, pero..." (técnica negación asertiva).

- Si no lo está en absoluto, expréselo también clara y asertivamente.

\subsection{Intervención asertiva}

En todo momento mostrar actitud Yo gano-Tú ganas.

- Con la normativa de referencia como apoyo, porque despersonaliza el conflicto. 
- La colaboración es la marca del servicio.

- Colaborar no significa incorporar demandas irracionales. Existen cuestiones que no se negocian.

- Si la otra parte elige lo contrario, es su decisión.

- Utilice los principios de la negociación YO GANO-TÚ GANAS:

- Separe a las personas del problema.

- Concéntrese en los intereses y no es las posiciones.

- Invente opciones en beneficio mutuo.

- Insista en utilizar criterios objetivos (normativa).

\subsection{Aplicación del análisis transaccional en la intervención asertiva}

El Análisis Transaccional es un modelo de personalidad que analiza las relaciones sociales y la comunicación entre las personas.

Según esta teoría nuestra estructura de la personalidad cuenta con tres estados:

1. El estado de padre. Cuando nos comunicamos desde el padre transmitimos normas, cómo se debe actuar, cuál es el funcionamiento. Existen roles en nuestra vida que corresponden a este estado. Se refiere a las introducciones que hemos ido incorporando de lo que se debe hacer y es válido en cada situación y lo que no, que en su momento fueron instalados en nosotros sin nuestro consentimiento. Son mandatos que nos inculcaron nuestros padres y demás adultos influyentes y son tomados de la cultura, de las tradiciones, de las normas, de los valores, del medio social en el que hemos crecido.

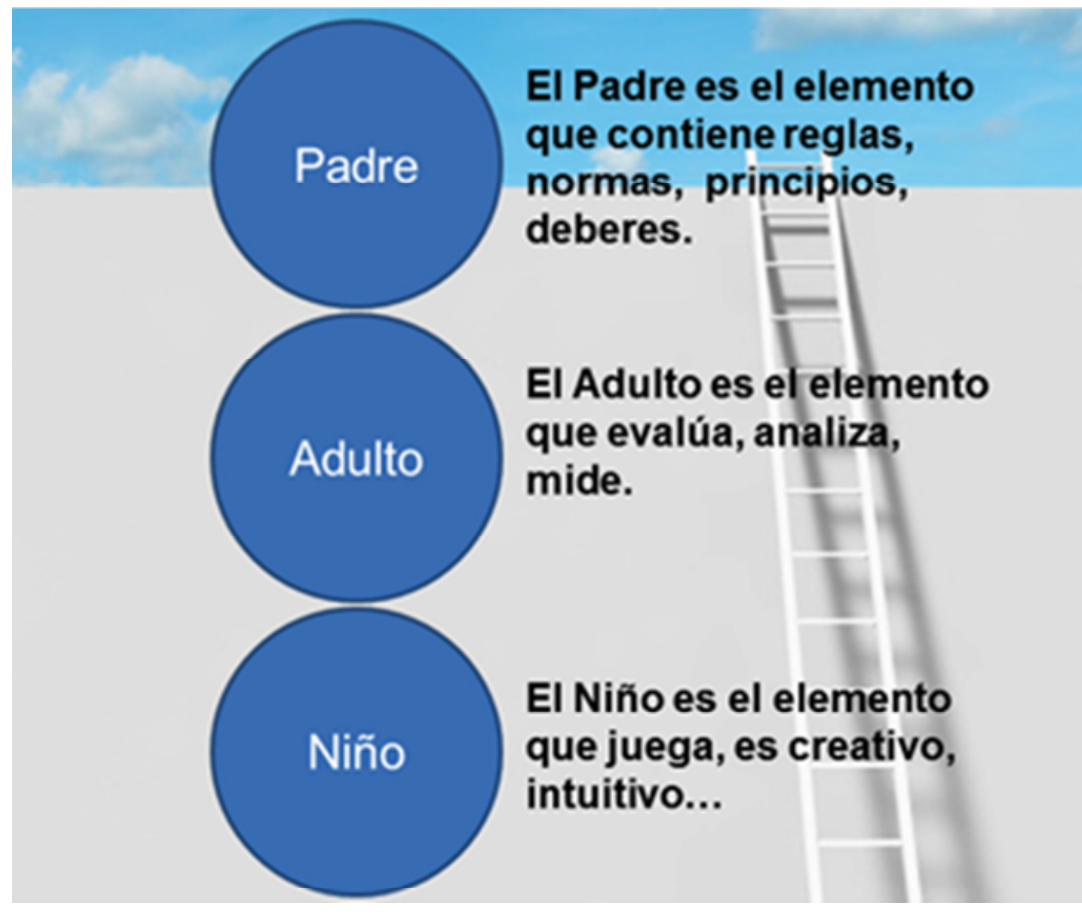


En este sentido, existen 2 tipos de PADRE:

a. Padre comprensivo: tiene la intención de guiar, controlar, encauzar desde una posición de respeto y protección.

b. Padre opresor: controla con postura de crítica destructiva y limitante, juicio, censura, y prepotencia.

2. El estado de adulto. Cuando nos comunicamos desde el adulto, transmitimos datos e información, de manera aséptica.

3. El estado de niño. Cuando nos comunicamos desde el niño, lo hacemos desde las emociones, tanto la alegría, el entusiasmo, la creatividad... como la rabia, el miedo, la impotencia, la frustración... Además del niño natural, distinguimos dos tipos de niño: el niño sumiso y el niño rebelde.

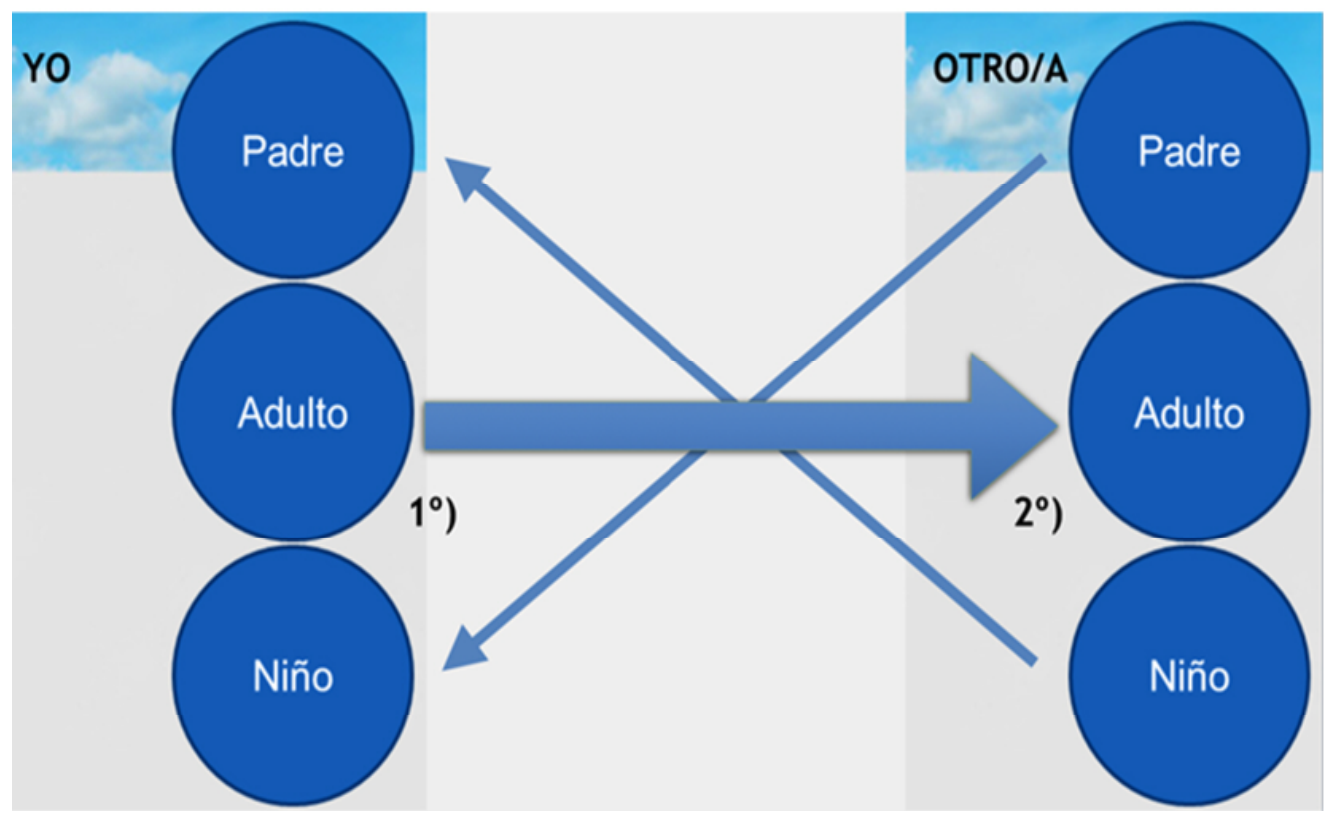

Es interesante darse cuenta de que se producen comunicaciones complementarias disfuncionales: padre opresor-niño (rebelde o sumiso), niño rebelde-niño rebelde, padre opresor-padre opresor, y resulta muy interesante observar cómo se van transformando estos estados en función del tono que toma la conversación. Estas transacciones complementarias tienen el efecto de reforzar los roles y la dinámica entre las partes.

Ante personas con comportamiento irracional, la comunicación asertiva en Análisis Transaccional consiste en 2 pasos:

a) Ponerse en el estado de adulto.

b) Hablar a la otra persona COMO SI tuviera el estado adulto activado. 


\section{Conclusiones}

No resulta nada fácil manejar el comportamiento irracional de las demás personas, concretamente los perfiles de personas difíciles que, con toda seguridad, son usuarias de los servicios de la Oficina del Defensor Universitario. Entre otras cosas porque nosotros también podemos llevar a cabo comportamientos de este tipo, muchas veces influenciados de manera inconsciente por el comportamiento irracional del otro y, en otras ocasiones, porque somos nosotros mismos quienes lo mostramos de entrada. No obstante, se hace necesario distinguir entre ocasiones puntuales o personas con estos patrones disfuncionales de relacionarse con los demás, personas difíciles que, probablemente, presenten algún trastorno mental, con o sin diagnosticar.

Para poder aplicar esta técnica se hace necesario abordar la conversación con distancia emocional. Se hace imperiosa la necesidad de incorporar:

- Recursos de autorregulación emocional.

- Técnicas de comunicación asertiva.

• Técnicas de negociación.

La buena noticia es que se trata de habilidades sociales y éstas, afortunadamente, se pueden aprender, si se entrenan. Oportunidades de entrenamiento no nos faltarán.

\section{Referencias}

AMERICAN PSYCHIATRIC ASSOCIATION (APA). (2002). Manual Diagnóstico y Estadístico de los Trastornos Mentales DSM-IV-TR. Barcelona: Masson.

Caballo, Vicente E. (1993). Manual de evaluación y entrenamiento de las habilidades sociales. Madrid: Siglo XXI de España Editores.

Haroa, Josep; Palacína, Concepció; Vilagutb, Gemma; Martínezb, Montse; Bernala, Mariola; Luquea, Inma; Codonyb, Miquel; Dolza, Montse; Alonsoby, Jordi; el Grupo ESEMeD-España (2005). Prevalencia de los trastornos mentales y factores asociados: resultados del estudio ESEMeD-España. Disponible en:

http://public-files.prbb.org/publicacions/e144ec9a-b14a-4cfd-bc18-56bf5c447ae6.pdf 